\title{
Excitation of THz surface waves in the conductor by a drag current generated by a focused femtosecond pulse
}

\author{
A.A. Frolov ${ }^{1}$, S.A. Uryupin ${ }^{2}$ \\ ${ }^{1}$ Joint Institute of High Temperature Physics, Russian Academy of Sciences, Moscow, 125412, Russia, frolov@ihed.ras.ru \\ ${ }^{2}$ P.N. Lebedev Physics Institute, Russian Academy of Sciences, Moscow, 119991, Russia, uryupin@sci.lebedev.ru
}

Surface waves (SW) are of interest because of their unusual physical properties and the possibility of the use of these waves for the diagnostics of surfaces, investigation of the properties of thin films and interfaces between various media, study of spectra of surface excited states, and signal transfer. The linear theory of SW excitation in conductors was developed in many works (see, e.g., [1]). Nonlinear methods of SW excitation are much less studied. At the same time, the experiment reported in [2] indicates the necessity of the development of a nonlinear theory of SW excitation. In application to the action of femtosecond pulses on conductors, we analyzed in [3] the possibility of SW exciting by a normally incident focused pulse. It is shown that the time-dependent ponderomotive force generates SW in a terahertz frequency range. Such a method of excitation can significantly increase the total energy of SW if a cylindrical lens is used to focus laser radiation [4].

In this work, we present the theory of another nonlinear mechanism of SW excitation caused by the generation of the drag current by a laser pulse which is focused by a cylindrical lens and is incident not at a right angle to the surface of a conductor. Since the effective frequencies of electron collisions for typical metals at room and higher temperatures are relatively high, the mechanism described below for the excitation of terahertz surface waves is more efficient than the ponderomotive mechanism.

Let the laser pulse of s-polarized radiation with amplitude of electric field $E_{0 L}$, frequency $\omega_{0}$ and duration $\tau$ be focused by a cylindrical lens in a line of length $L_{y}$ and the transverse dimension $L_{x}$ falls on the boundary of the conducting medium at an angle $\alpha$ relative to the normal. The laser field penetrating into conductor generates a slowly varying drag current along the surface of the conductor and provides the ponderomotive action on electrons, which generate low-frequency currents along and across the surface. To determine nonlinear currents $\mathbf{j}(\mathbf{r}, t)$, we use the equation

$$
\begin{gathered}
\frac{\partial}{\partial t} \mathbf{j}(\mathbf{r}, t)+v_{s} \mathbf{j}(\mathbf{r}, t)= \\
=\frac{\omega_{p}^{2}}{4 \pi e}\left[e \mathbf{E}(\mathbf{r}, t)-\nabla W(\mathbf{r}, t)+\mathbf{e}_{\mathrm{x}} \frac{2 v_{h}}{c} W(\mathbf{r}, t) \sin \alpha\right],
\end{gathered}
$$

where $\omega_{\mathrm{p}}$-the plasma frequency, $v_{h(s)}$-the frequency of electron collisions in the high (low)-frequency field, $e$ is the elementary charge, $W(\mathbf{r}, t)$-the ponderomotive potential in the conductor, $c$-speed of light. Constitutive equation (1) and Maxwell's equations for the electric $\mathbf{E}(\mathbf{r}, t)$ and magnetic $\mathbf{B}(\mathbf{r}, t)$ fields

$$
\begin{gathered}
r o t \mathbf{B}=\frac{1}{c} \frac{\partial}{\partial t} \mathbf{D}+\frac{4 \pi}{c} \mathbf{j}, \operatorname{rot} \mathbf{E}=-\frac{1}{c} \frac{\partial}{\partial t} \mathbf{B}, \\
\mathbf{D}(\mathbf{r}, t)=\int_{-\infty}^{+\infty} \frac{d \omega}{2 \pi} e^{-i \omega t} \varepsilon_{0}(\omega) \mathbf{E}(\mathbf{r}, \omega),
\end{gathered}
$$

where $\mathbf{D}(\mathbf{r}, t)$ is the electric displacement, $\varepsilon_{0}$ - contribution to the permittivity of bound electrons and lattices, make it possible to describe a low-frequency electromagnetic field in the conductor. In Eq. (1), the term proportional to the gradient of $W(\mathbf{r}, t)$ is due to the ponderomotive action of laser radiation and the term with $v_{h}$ corresponds to the effect of the drag current. The effect of the ponderomotive action can be neglected under the conditions

$$
v_{h} \tau \gg 1, \quad v_{h} \tau>\left(L / L_{x}\right) \operatorname{ctg} \alpha,
$$

where $L=c \tau$ is the longitudinal size of laser pulse. Under conditions (3), the drag current is the main reason for the generation of surface waves. Because of the oblique incidence, a laser pulse generates SW propagating in the direction of the drag current and in the opposite direction, which have different parameters.

Spectral and energy parameters of the surface waves are calculated. The energy of the SW per unit frequency interval $\mathrm{d} \omega$ has the form

$$
\frac{d W^{ \pm}(\Omega, \alpha)}{d \Omega}=\frac{e^{2} E_{0 L}^{4} L_{x}^{2} L^{2} L_{y}}{4 \sqrt{2} m^{2} c^{4}} \frac{\left(v_{h} \tau\right)^{2}}{\left(v_{s} \tau\right)^{1 / 2}} \frac{I^{ \pm}(\Omega, \alpha)}{\omega_{p}^{5} \tau^{5}},
$$

where

$$
\begin{aligned}
& I^{ \pm}(\Omega, \alpha)=\Omega^{5 / 2} \sin ^{2} \alpha \cos ^{2} \alpha \times \\
& \times \exp \left\{-\frac{\Omega^{2}}{2}\left(1+\frac{L_{x}^{2}}{L^{2}} \frac{1 \mp \sin \alpha}{1 \pm \sin \alpha}\right)\right\},
\end{aligned}
$$

$\Omega=\omega \tau$-dimensionless frequency, $m$-electron mass, $W^{+}, W$ - energy of the SW in the direction of the drag current and in the opposite direction. The frequency distribution of SW given by Eq. (5) has a maximum at

$$
\omega_{\mathrm{m}}^{ \pm}=\sqrt{\frac{5}{2}} \frac{1}{\tau}\left(1+\frac{L_{x}^{2}}{L^{2}} \frac{1 \mp \sin \alpha}{1 \pm \sin \alpha}\right)^{-1 / 2} .
$$

The maximum in the spectrum for SW, propagating in the direction of the drag current, shifts to the highfrequency region with increasing incidence angle $\alpha$ (Fig. 1). In contrast to this for SW propagating in the direction opposite to the drag current, the maximum in the spectrum shifts to the region of low frequencies with increasing incidence angle $\alpha$ (Fig. 2).

The integration of Eq. (4) with respect to the frequency gives the total energy of surface waves:

$$
W^{ \pm}(\alpha)=\frac{3}{2^{7 / 4}} \Gamma\left(\frac{3}{4}\right) \frac{\left(v_{h} \tau\right)^{2}}{\left(v_{s} \tau\right)^{1 / 2}} \frac{e^{2} E_{0 L}^{4} L_{y}}{4 m^{2} c^{4}} \frac{w^{ \pm}(\alpha)}{k_{p}^{5} L},
$$




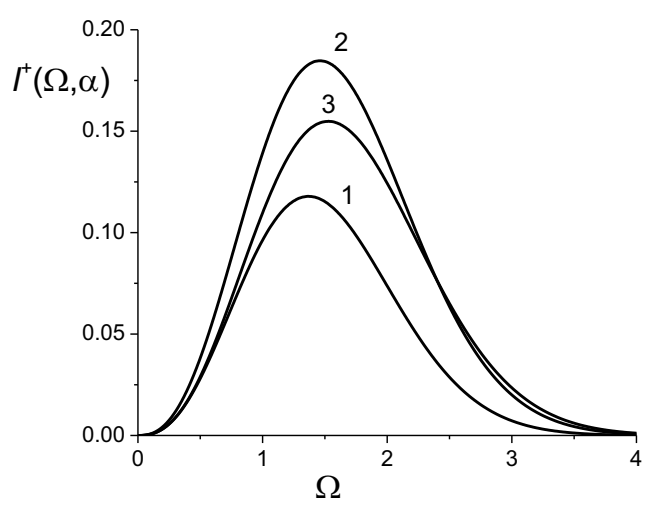

Fig. 1. The dependence of the energy of the SW propagating in the direction of the drag current on the frequency $\omega \tau$ for different angles of incidence $\alpha$ : (1) $\alpha=\pi / 6$, (2) $\alpha=\pi / 4$, (3) $\alpha=\pi / 3$ for $L=L_{x}$

where $\Gamma(x)$ is the Gamma function, $k_{p}=\omega_{\mathrm{p}} / c$

$$
w^{ \pm}(\alpha)=\frac{L_{x}^{2}}{4 L^{2}} \sin ^{2} 2 \alpha\left(1+\frac{L_{x}^{2}}{L^{2}} \frac{1 \mp \sin \alpha}{1 \pm \sin \alpha}\right)^{-7 / 4} \text {. }
$$

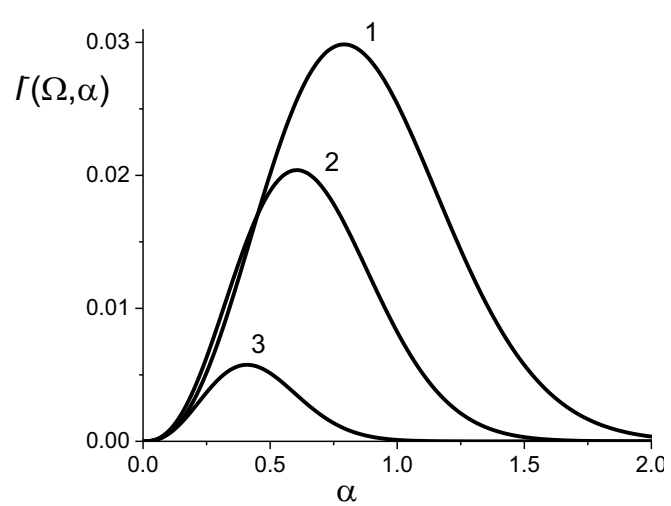

Fig. 2. The dependence of the energy of the SW propagating in the direction opposite to the drag current on the frequency $\omega \tau$ for different angles of incidence $\alpha$ : (1) $\alpha=\pi / 6$, (2) $\alpha=\pi / 4$, (3) $\alpha=\pi / 3$ for $L=L_{x}$

The dependence of the energy of the SW propagating in the direction of the drag current (8) on the angle $\alpha$ is shown in Fig. 3. In the case of a tightly focused pulse, $L_{x}<<L$, the function $w^{+}(\alpha)$ in Eq. (8) has a maximum of $w^{+}=\left(L_{x} / 2 L\right)^{2}$ at $\alpha=\pi / 4$. If $L_{x}=L, w^{+}(\alpha)$ has the maximum $w^{+} \approx 0.195$ at $\alpha \approx 49.6^{\circ}$. Finally, for a poorly focused pulse when $L_{x}>>w^{+}(\alpha)$ has a largest value of maximum $w^{+} \approx 1.2$ at $\alpha \approx \pi / 2-4 L /\left(L_{x} \sqrt{ } 3\right)$. Thus with an increase of the laser pulse transverse size $L_{x}$ the energy of SW propagating in the direction of the drag current increases.

The dependence of the energy of the SW propagating in the direction opposite to the drag current, on the angle of incidence of the laser pulse $\alpha$ is shown in Fig. 4. As follows from Eq (8) and Fig. 4 with an increase in the transverse size, the energy of the SW propagating in the direction opposite to the drag current decreases.

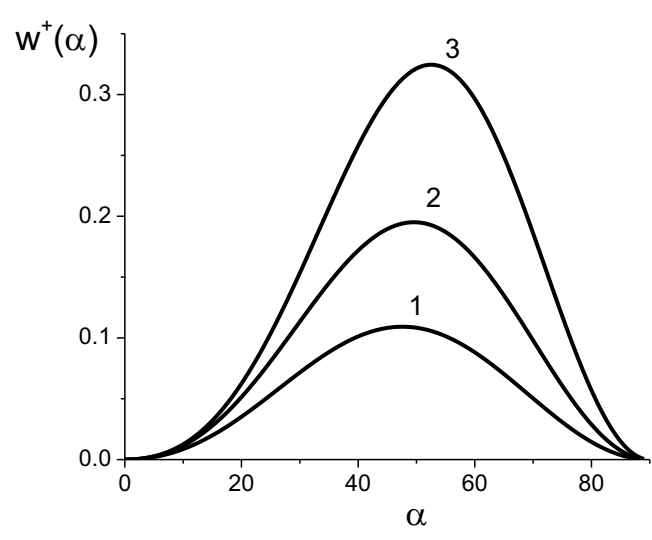

Fig. 3. The dependence of the total energy of the SW propagating in the direction of the drag current on the angle of incidence of the laser pulse $\alpha$ : (1) $\left(L_{x} / L\right)^{2}=1 / 2,(2)\left(L_{x} / L\right)^{2}=1,(3)\left(L_{x} / L\right)^{2}=2$

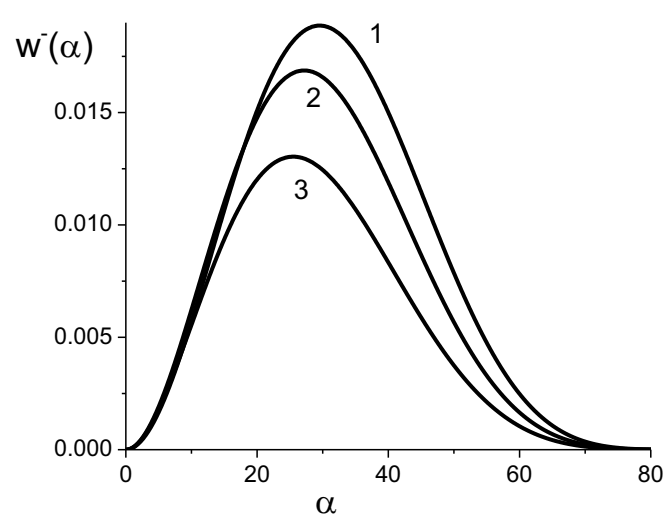

Fig. 4. The dependence of the total energy of the SW propagating in the direction opposite of the drag current on the angle of incidence of the laser pulse $\alpha$ : $(1)\left(L_{x} / L\right)^{2}=1 / 2,(2)\left(L_{x} / L\right)^{2}=1$, (3) $\left(L_{x} / L\right)^{2}=2$

The energy of SW propagating in the direction of the drag current greatly exceeds the SW energy propagating in the opposite direction.

The work supported by RFBR, project N17-02$00648 \mathrm{a}$.

\section{References}

1. Maier S.A. Plasmonics: Fundamentals and Applications. Springer, Berlin, 2007; R\&C Dynamics, Moscow, 2011, P. 22.

2. Ionin, A.A., Kudryashov, S.I., Seleznev, L.V., Sinitsin, D.V., Emel'yanov, V.I. Nonlinear regime of the excitation of a surface electromagnetic wave on the silicon surface by an intense femtosecond laser pulse // JETP Lett. 2013. V. 97, No. 3. P. 121-126.

3. Uryupin, S.A., Frolov, A.A. Excitation of surface waves by a short laser pulse in a conductor // Quantum Electron. 2013. V. 43. No. 12. P. 1132-1138.

4. Uryupin, S.A., Frolov, A.A. Generation of surface waves and low-frequency radiation under exposure of a conductor to a laser pulse focused by a cylindrical lens // Quantum Electron. 2014. V. 44. No. 9. P. 866-872. 